\title{
Pexidartinib: first approved systemic therapy for patients with tenosynovial giant cell tumor
}

\author{
Hans Gelderblom *,1 (iD) \& Michiel van de Sande ${ }^{1}$ \\ ${ }^{1}$ Leiden University Medical Center, Leiden, Netherlands \\ *Author for correspondence: Tel.: +31 71526 3486; a.j.gelderblom@lumc.nl
}

Pexidartinib is an orally administered small molecule tyrosine kinase inhibitor. Phase III ENLIVEN study results provided clinical evidence for US FDA approval for treatment of adult patients with symptomatic tenosynovial giant cell tumor associated with severe morbidity or functional limitations and not amenable to improvement with surgery. Recommended dosage is $400 \mathrm{mg}$ orally twice daily on an empty stomach. Long-term follow-up in pooled analyses showed increased response rates compared with those observed in ENLIVEN. Patients on pexidartinib also experience meaningful improvements in range of motion. Side effects associated with pexidartinib are generally manageable; however, there is a risk of potentially lifethreatening mixed or cholestatic hepatotoxicity and pexidartinib has a Risk Evaluation and Mitigation Strategy (REMS) program to ensure appropriate monitoring.

First draft submitted: 26 May 2020; Accepted for publication: 23 June 2020; Published online: 23 July 2020

Keywords: CSF1 • CSF-1R inhibitor • neoplasm • patient-reported outcomes • pexidartinib • RECIST • safety • tenosynovial giant cell tumor $\bullet$ TGCT • tumor volume score

Tenosynovial giant cell tumor (TGCT) is a rare, nonmalignant neoplasm that can be locally aggressive [1]. In the USA, the estimated annual incidence of TGCT is 11 per million in the overall population [2], and studies from the European Union provide an estimate of approximately 39-50 per million person-years [3,4]. Individuals with TGCT have lesions that typically arise from the synovium of joints, bursae or tendon sheaths $[1,5]$. Lesions can present as single nodules, also known as localized TGCT (L-TGCT), which is most frequently seen in the fingers [6]. TGCT may also occur as multiple nodules, also known as diffuse-type TGCT (D-TGCT), most commonly occurring in the knee but also observed in the hip, ankle, wrist, shoulder or elbow [6]. In 2013, the WHO reorganized the types of TGCT as L-TGCT and D-TGCT, replacing the terms giant cell tumor of the tendon sheath (GCT-TS) and pigmented villonodular synovitis (PVNS), respectively [5]; however, the former terms GCT-TS and PVNS are sometimes used in clinical practice and in the literature [5].

Patients who have TGCT generally experience increased pain and impaired daily functioning compared with the general population, with poorer quality of life reported for patients with D-TGCT compared with L-TGCT [7]. In a crowdsourcing study of a TGCT social media group, participants were invited to complete an e-survey to determine the impact of TGCT on daily functioning and overall quality of life; patients with diffuse type, recurrent disease or at least two surgeries had the worst functional and quality of life outcomes [7]. This study also reported that patients with TGCT were limited in their ability to fully perform employment duties (13\% for L-TGCT and 11\% for D-TGCT) and were unable to perform sports activities (58\% for L-TGCT and $64 \%$ for D-TGCT) [7]. In the prospective observational study TGCT Observational Platform Project (TOPP; $\mathrm{n}=183$ ), participants with TGCT reported decreased mobility, problems with self-care, problems performing usual activities, pain or discomfort and above all anxiety or depression associated with TGCT compared with the general population [8].

Current clinical practice guidelines recommend evaluation and management by a multidisciplinary team and surgical excision as first-line treatment for resectable disease [9-11]. For TGCT, the current standard of care is to surgically resect the tumor as completely as possible [12]. D-TGCT is typically more difficult to manage surgically than L-TGCT [6,13]. Complete resection, particularly difficult, to sometimes impossible for patients with D-TGCT,

Future Medicine 
is associated with postoperative comorbidities such as continuing pain, swelling, joint stiffness and instability [5]. Additionally, tumors often recur, especially with D-TGCT where the recurrence-free survival rate at 5 years is estimated at about $64 \%$ for primary disease and $25 \%$ for recurrent disease [14-17]. Many patients undergo multiple surgeries [18], which may result in significantly more and long-term morbidity, with a very high risk for disease progression [5]. In the USA, the approved agent pexidartinib is the first systemic therapy available for treatment of patients with TGCT [19]. Imatinib and nilotinib are approved for other indications, but in some countries they have been used off-label to treat TGCT [5]. Although radiotherapy is not standard (because of side effects including stiffness, chondrolysis, osteonecrosis and most importantly risk for secondary malignancies), it may be considered as a treatment option in recurrent nonresectable cases [5,10].

TGCT lesions are driven by an overexpression of CSF1 present in a minority of neoplastic cells within the tumor, which leads to tumor formation via the recruitment and accumulation of inflammatory cells [20]. Pexidartinib is a novel small molecule inhibitor of the CSF1 receptor (CSF1R) and c-KIT receptor, and is a more potent inhibitor of CSF1R than imatinib or nilotinib [14].

Pexidartinib is the first approved systemic therapy for patients with TGCT; it is currently available in the USA and has an accompanying Risk Evaluation and Mitigation Strategy (REMS) program [19]. It is approved for the treatment of adult patients with symptomatic TGCT associated with severe morbidity or functional limitations and not amenable to improvement with surgery [19]. In the USA, the recommended dosage is $400 \mathrm{mg}$ orally twice daily on an empty stomach, at least $1 \mathrm{~h}$ before or $2 \mathrm{~h}$ after a meal or snack [19]. The prescribing information for pexidartinib includes a black box warning for serious and potentially fatal liver injury, although these events occur in a small minority of patients [19]. Pexidartinib is recommended for patients with TGCT in the National Comprehensive Cancer Network 2019 Clinical Practice Guidelines in Oncology for soft tissue sarcoma, with a category 1 recommendation [9].

\section{Pharmacodynamics \& pharmacokinetics}

In TGCT, a neoplastic clone aberrantly expresses CSF1, often due to $t(1 ; 2)$ CSF1:COL6A3 translocation [20]. This dysregulated CSF1 attracts CSF1R-bearing mononuclear cells that form the bulk of the tumor; the majority of cells in TGCT tumors are reactive, nonneoplastic cells, with a minority of the cells being neoplastic [14,20].

Pexidartinib was designed to access the autoinhibited state of CSF1R through direct interactions with juxtamembrane residues embedded in the ATP-binding pocket [14]. Pexidartinib binds CSF1R in its autoinhibited state, stabilizing the receptor in an inactive form and preventing ligand (CSF1) binding (Figure 1) [14]. Pexidartinib inhibits CSF1R on infiltrating macrophages, which prevents binding of CSF1 and disrupts the autocrine and paracrine loops driving tumor growth [20]. This was demonstrated in a mouse xenograft model of malignant peripheral sheath tumors in which pexidartinib suppressed tumor growth through depletion of macrophages [21]. In another study, pexidartinib shifted the polarization of tumor-associated macrophages to an M1-like phenotype [22].

An exact experimental model of TGCT does not exist; however, a mouse model of collagen-induced arthritis shares several pathologic features with TGCT [14]. In that model, oral administration of pexidartinib $10 \mathrm{mg} / \mathrm{kg}$ significantly delayed the progression and reduced the severity of disease, including inhibition of severe bone erosion, cartilage destruction, synovitis and pannus formation [14].

Following oral administration, pexidartinib is rapidly absorbed, with a median time to maximum plasma concentration $\left(\mathrm{T}_{\max }\right.$ ) of approximately $2.5 \mathrm{~h}$ and a mean half-life of approximately $27 \mathrm{~h}$ [19]. When pexidartinib is administered as a single oral dose, the exposure increased linearly over a dose range of 200-2400 mg. At steady state, at a dosage of $400 \mathrm{mg}$ twice daily, the mean (standard deviation [SD]) maximum serum concentration $\left(\mathrm{C}_{\max }\right)$ was $8625(2746) \mathrm{ng} / \mathrm{ml}$; the mean $(\mathrm{SD})$ area under the concentration-time curve from $0-12 \mathrm{~h}\left(\mathrm{AUC}_{0-12 \mathrm{~h}}\right)$ was $77,465(24,975) \mathrm{ng} / \mathrm{h} / \mathrm{ml}$ [19]. Although the clinical studies had a 2-week loading dose of $1000 \mathrm{mg}$ per day, an exposure-response analysis supported the administration of $800 \mathrm{mg} /$ day without a loading dose for patients with TGCT [23].

Pexidartinib pharmacokinetics can be influenced by food intake. Studies in healthy subjects have demonstrated that administration of pexidartinib with a high-fat ( $\sim 50 \%$ of total calories from fat) meal led to an approximate doubling in pexidartinib exposure when compared with the fasted state. When pexidartinib was administered with a low-fat meal ( $\sim 25 \%$ of calories from fat), exposure increased by approximately $60 \%$. When pexidartinib was administered with a high-fat meal, median time to $T_{\max }$ was delayed by approximately $2.5 \mathrm{~h}$, while administering pexidartinib with a low-fat meal delayed $T_{\max }$ by $1.5 \mathrm{~h}$. These findings highlight the importance of taking pexidartinib on an empty stomach at least $1 \mathrm{~h}$ before or $2 \mathrm{~h}$ after a meal or snack [19]. 
(A)

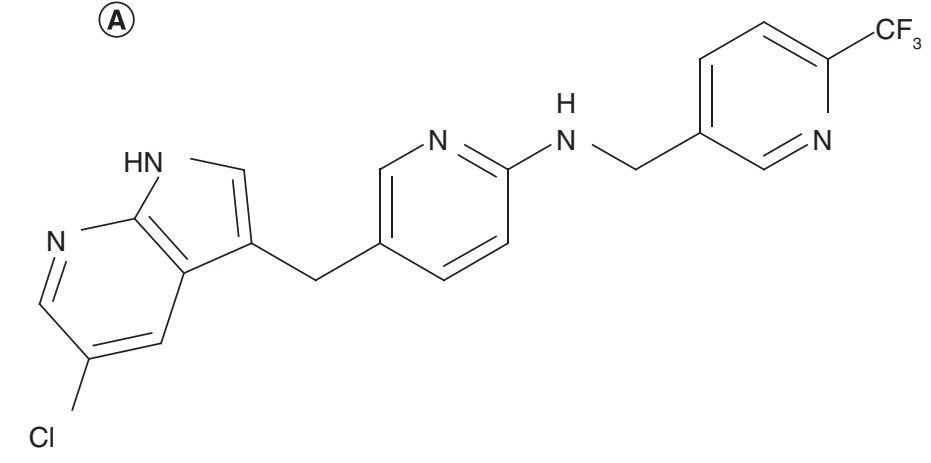

$\cdot \mathrm{HCl}$

(B)

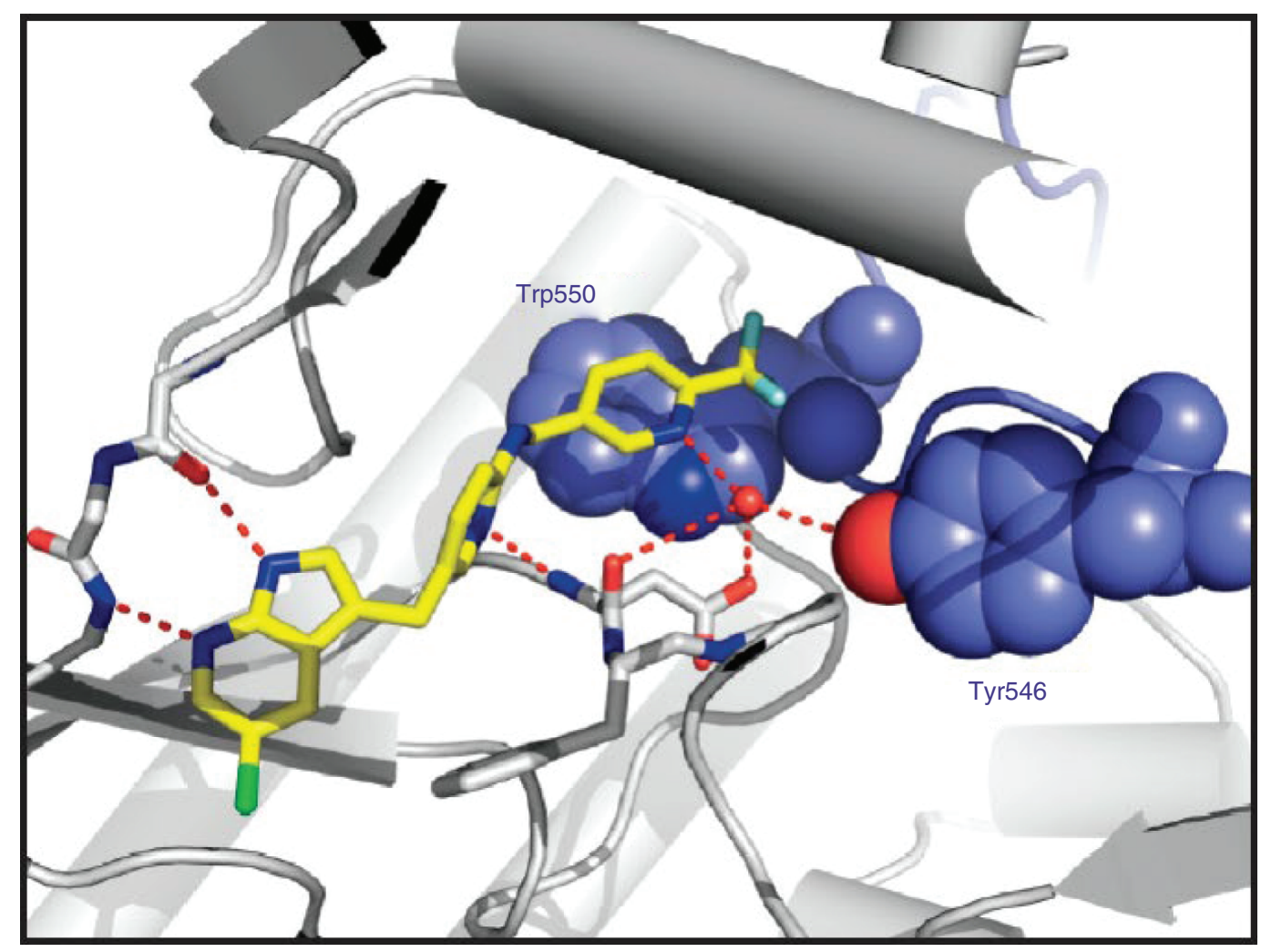

Figure 1. Pexidartinib and the CSF1 receptor. (A) Chemical structure of pexidartinib and (B) conformation-specific inhibition of CSF1R. Reproduced in part with permission from [14] (C) Massachusetts Medical Society (2015). Reprinted with permission from Massachusetts Medical Society.

A pharmacokinetic model that included healthy volunteers and patients from a Phase I study and the Phase III ENLIVEN study in patients with TGCT identified no clinically meaningful effects of pexidartinib exposure for demographic characteristics (such as race, healthy volunteers vs patients) or renal and hepatic functional parameters [24]. Similarly, a population pharmacokinetics analysis showed similar exposures between Asian and non-Asian patients [25].

\section{Clinical studies of pexidartinib in TGCT}

Pexidartinib was examined in TGCT in a two-part Phase I study [14]. In part 1, a dose-escalation study evaluated the safety and pharmacokinetics of pexidartinib in patients with solid tumors. Part 2 was a multicenter, multi-cohort 
(A)

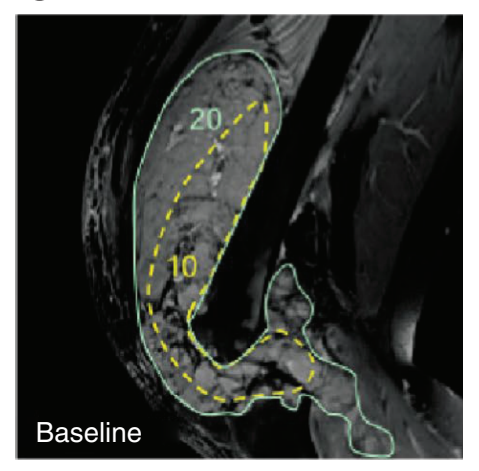

(B)

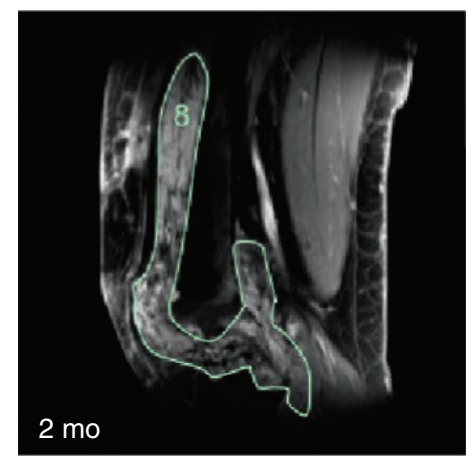

(C)

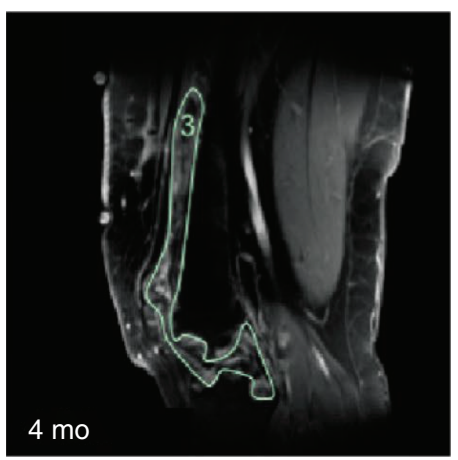

Figure 2. Efficacy assessment by tumor volume score. (A) The solid green line delineates the perimeter of the tumor in the baseline image. Follow-up images (B\&C) are used to determine the tumor volume score. The dashed yellow line in (A) shows the estimated maximum volume of the normal synovial cavity, against which the tumor volume score is referenced. Note that the tumor volume score is based on all the slices through the entire synovial cavity and not just the areas depicted in the single mid-sagittal slices.

Reproduced in part with permission from [14] ( Massachusetts Medical Society (2015). Reprinted with permission from Massachusetts Medical Society.

extension study designed to examine safety and efficacy of pexidartinib in patients with select different tumors including those with histologically confirmed TGCT with progression within the past year and with disease that was recurrent, inoperable or required extensive surgery for resection.

In part 1, a total of 41 patients received pexidartinib 200, 300, 400, 600, 900, 1000 or $1200 \mathrm{mg}$ daily [14]. Among the 41 patients, five (12\%) had a total of eight dose-limiting adverse events, which included one patient at $300 \mathrm{mg}$ (increased international normalized ratio and hematuria), one patient at $600 \mathrm{mg}$ (hyponatremia), two patients at $1200 \mathrm{mg}$ (one patient had an increase in AST level and one had anemia, neutropenia and syncope) and one patient at $1000 \mathrm{mg}$ (increase in AST level). The maximum tolerated dose was $1000 \mathrm{mg} /$ day. Eleven (27\%) patients had at least one grade $\geq 3$ event considered possibly or probably related to treatment. Pharmacokinetics analysis showed that, in general, maximum concentrations and AUC from 0-24 h $\left(\mathrm{AUC}_{0-24}\right)$ exposures increased with increasing dose. At the time of the original report, 23 of the 41 patients with advanced TGCT had enrolled in the dose extension study; the mean age was 46 years and $15(65 \%)$ patients had disease in the knee. All 23 patients reported adverse events and treatment-related adverse events. Response was measured using Response Evaluation Criteria in Solid Tumors version 1.1 (RECIST). By RECIST, 12 of 23 patients had a partial response (overall response rate $[\mathrm{ORR}]=52 \%$; 95\% CI: 32-73) and seven of $23 \mathrm{had}$ stable disease; median progression-free survival was not reached [14]. Efficacy was also evaluated using a novel response criterion, tumor volume score (TVS). TVS is a modification of the rheumatoid arthritis magnetic resonance imaging (MRI) score and the whole-organ MRI score, which are commonly used in arthritis. Measurement of the tumor responses in TGCT is challenging because of the irregular shape of the tumor and heterogeneous contrast between the tumor and its surrounding structure [14,26]. TVS is a semiquantitative scoring method developed specifically for assessing TGCT (Figure 2) [14]. The score calculates tumor volume as a percentage of the entire synovium, with standardization to the synovial or tenosynovial cavity. In the dose extension study, 14 patients were evaluable for TVS; 11 patients had a partial response and three had stable disease [14]. Histological analysis of pretreatment and post-treatment tumor biopsies from one patient with TGCT showed decreases in the cellularity and reduced numbers of pigment-laden macrophages 2 weeks after treatment, consistent with the targeting and depletion of macrophages by pexidartinib [14].

The ENLIVEN study was the first randomized, placebo-controlled Phase III study in patients with TGCT [27]. Eligible patients had histologically confirmed, advanced, symptomatic TGCT with measurable tumors of at least $2 \mathrm{~cm}$ per RECIST for which surgical resection was associated with a potential for worsening of functional limitation or severe morbidity [27]. This study had two parts: a double-blind, randomized, placebo-controlled study (part 1), followed by an open-label crossover study of pexidartinib for all patients (part 2). In part 1, patients received pexidartinib $1000 \mathrm{mg}$ (or matching placebo) split twice a day for 2 weeks, followed by $800 \mathrm{mg}$ split twice per day 


\begin{tabular}{|c|c|c|c|c|}
\hline $\begin{array}{l}\text { End point } \\
\text { RECIST }\end{array}$ & $\begin{array}{l}\text { ENLIVEN randomized } \\
\left(1000 \mathrm{mg} / \text { day }^{\dagger}\right) \\
\mathrm{n}=61\end{array}$ & $\begin{array}{l}\text { ENLIVEN crossover } \\
\left(800 \mathrm{mg} / \text { day }^{\dagger}\right) \\
\mathrm{n}=30\end{array}$ & $\begin{array}{l}\text { PLX108-01 TGCT cohort } \\
\left(1000 \mathrm{mg} / \text { day }^{\dagger}\right) \\
\mathrm{n}=39\end{array}$ & $\begin{array}{l}\text { Pooled } \\
n=130\end{array}$ \\
\hline CR & $18(30)$ & $8(27)$ & $8(21)$ & $34(26)$ \\
\hline PR & $20(33)$ & $10(33)$ & $14(36)$ & $44(34)$ \\
\hline Not evaluable & $9(15)$ & $4(13)$ & $12(31)$ & $25(19)$ \\
\hline \multicolumn{5}{|l|}{ TVS } \\
\hline ORR & $40(66)$ & $20(67)$ & $24(62)$ & $84(65)$ \\
\hline $\mathrm{CR}$ & $5(8)$ & 1 (3) & $8(21)$ & $14(11)$ \\
\hline PR & $35(57)$ & $19(63)$ & $16(41)$ & $70(54)$ \\
\hline \multicolumn{5}{|c|}{$\begin{array}{l}\text { Data represented as } n(\%) \text {. } \\
\text { † Starting dose. }\end{array}$} \\
\hline
\end{tabular}

for 22 weeks. In part 2, patients either maintained their part 1 dose of pexidartinib or crossed over from placebo. ENLIVEN enrolled and randomized 120 patients to receive at least one dose of pexidartinib (61 patients) or placebo (59 patients) in part 1; 30 patients crossed over from placebo to receive pexidartinib in part 2 resulting in a total of 91 patients receiving pexidartinib in ENLIVEN. The most common disease site was the knee (73 [61\%] of 120 patients); 63 (53\%) had at least one previous surgery for TGCT, and 11 (9\%) had previously received a tyrosine kinase inhibitor (TKI; imatinib or nilotinib). At 25 weeks, the ORR based on RECIST was 39\% (95\% CI: $28-52$ ) in the pexidartinib group versus $0 \%$ (95\% CI: $0-6$ ) in the placebo group ( $\mathrm{p}<0.0001$ ); the ORR based on TVS was $56 \%$ (95\% CI: 43-68) in the pexidartinib group versus $0 \%$ (95\% CI: $0-6)$ in the placebo group $(\mathrm{p}<0.0001)$. The median duration of response was not reached by either RECIST (range, 0-25 months) or TVS (range, 0-28 months) with a median duration of follow-up of 22 months [27], demonstrating robust response to treatment.

In addition to TVS, ENLIVEN included other TGCT-specific end points examining patient symptoms and functional outcomes, such as range of motion, Patient-Reported Outcomes Measurement Information System (PROMIS), patient-reported stiffness and pain response [27,28]. There was a significant improvement from baseline in range of motion in patients randomized to pexidartinib versus placebo at week $25(15.1 \mathrm{vs} 6.2 \%$; $\mathrm{p}=0.0043)$. The PROMIS-physical function (PROMIS-PF) scale addresses symptoms of immobility and includes scales for both lower and upper extremity tumors; a 2-3-point change in PROMIS-PF represents a clinically meaningful difference large enough to affect a patient's physical functioning [27]. Patients in the pexidartinib group had clinically meaningful improvements from baseline in PROMIS-PF scores at week 25, while patients in the placebo group reported no improvement (4.1 vs $-0.9 ; \mathrm{p}=0.0019)$. Patients who received pexidartinib also experienced a significant improvement from baseline in worst stiffness $(-2.5$ vs $-0.3 ; \mathrm{p}<0.0001)$ compared with placebo [27]. In subgroup analysis, pexidartinib reduced tumor size and improved pain response consistently in patients with or without prior surgery or prior radiation, with localized or diffuse TGCT, and irrespective of tumor site (lower vs upper extremity, large vs small joints) [29]. Limitations to these results include missing data at week 25.

Long-term follow-up with a median treatment duration of 19 months demonstrated increased tumor response rate with prolonged treatment [30-32]. A pooled analysis of data from ENLIVEN and the Phase I study demonstrated prolonged responses with pexidartinib and increased response rates with long-term treatment. At 19 months followup, the response rate was even greater: the ORR was $60 \%$ by RECIST and $65 \%$ by TVS, with patients shifting from stable disease to partial or complete response between these timepoints (Table 1) [30-32].

Pexidartinib has also been tested in patients with malignant tumor types, with limited efficacy. No efficacy was shown with pexidartinib compared with historic controls in a Phase II open-label study in patients with recurrent 
glioblastoma or in a Phase I/II study in combination with radiation therapy and temozolomide in patients with newly diagnosed glioblastoma [33,34]. In combination with paclitaxel in advanced solid tumors, there was one complete response and five partial responses in 38 evaluable patients [35]. Pexidartinib has shown some activity in combination with sirolimus for patients with malignant peripheral nerve sheath tumor [36], in combination with binimetinib or PLX9486 for advanced gastrointestinal stromal tumor $[37,38]$ and as monotherapy for pediatric tumors including neurofibromatosis type I related plexiform neurofibromas [39].

\section{Safety}

In part 1 of the ENLIVEN study, treatment-emergent adverse events were reported in 60 (98\%) of 61 patients who received pexidartinib and $55(93 \%)$ of 59 patients who received placebo [27]. The most common $(\geq 20 \%)$ adverse events of any grade in part 1 of ENLIVEN were hair color changes (67\%), fatigue (54\%), AST increase (39\%), nausea (38\%), ALT increase (28\%), dysgeusia (25\%), arthralgia (23\%), diarrhea (20\%) and vomiting (20\%). Common grade 3 or 4 adverse events occurring at a higher rate in the pexidartinib group than in the placebo group were increased AST (10 vs 0\%), increased ALT (10 vs 0\%), increased ALP (7 vs 0\%) and hypertension (5 vs 0\%). Eight (13\%) of 61 patients discontinued pexidartinib due to adverse events; seven of these discontinuations were related to liver adverse events [27].

In a pooled analysis of pexidartinib studies, the most frequent adverse events were hair color changes $(75 \%)$, followed by fatigue (61\%), nausea (47\%), arthralgia (39\%), diarrhea (32\%), increased AST (31\%) and dysgeusia (30\%; Table 2) [31,32]. Long term, the overall safety profile of pexidartinib was similar to previous findings [30-32]. In a randomized, placebo-controlled three-period crossover study conducted in healthy subjects, no impact on QTcF was observed for plasma concentrations up to $30 \mu \mathrm{g} / \mathrm{ml}$ and supratherapeutic doses (2.5-fold the predicted steady-state $\mathrm{C}_{\max }$ ) did not cause clinically relevant electrocardiogram effects [40].

\section{Hepatic adverse reactions}

The hepatic safety profile of pexidartinib consisted largely of frequent, dose-dependent, generally low-grade aminotransferase elevations related to CSF1R inhibition $[31,32,41]$. Liver enzyme abnormalities are a known class effect of CSF1R antagonists due to depletion of Kupffer cells [42,43]. However, hepatic adverse reactions associated with pexidartinib also included uncommon and idiosyncratic, yet potentially life-threatening, mixed or cholestatic hepatotoxicities [31,32,41]. In TGCT studies, five of 140 (3.6\%) patients had serious hepatic adverse reactions, all within the first 2 months of treatment. All cases were reversible with discontinuation of pexidartinib: four resolved within 2 months, and one case of ductopenia and severe cholestasis resolved after 7 months. No new safety signals of serious mixed or cholestatic hepatotoxicity occurred in long-term follow-up [31,32,41]. Four of the five serious hepatic adverse reactions occurred with the $1000 \mathrm{mg} /$ day dose; the other occurred at the $800 \mathrm{mg} / \mathrm{day}$ dose [41]. Across the clinical program, there were two irreversible cases of cholestatic liver injury. One patient died with advanced cancer and ongoing liver toxicity and one patient required a liver transplant [44].

\section{Clinical approach to managing pexidartinib safety}

Given the slow-growing nature of TGCT and the risk of serious and potentially life-threatening hepatotoxicity, careful consideration must be taken when assessing the benefit:risk. The results of ENLIVEN demonstrate that pexidartinib achieved sustained tumor response and improved mobility in patients with TGCT [27]. In light of the observed hepatic adverse reactions, careful monitoring of liver function is advised, particularly in the first 2 months of treatment. Informed discussions on the potential risks of hepatotoxicity should occur between patients and their healthcare team. Per prescribing guidelines in the USA, pexidartinib should be avoided in patients with pre-existing increased serum transaminases, total bilirubin or direct bilirubin (greater than the upper limit of normal) or active liver or biliary tract disease including increased ALP [19]. Liver tests (ALT, AST, total and direct bilirubin, ALP and GGT) should be monitored prior to initiation of pexidartinib, weekly for the first 8 weeks, every 2 weeks for the next month and every 3 months thereafter [19]. The currently recommended dosage of pexidartinib in the USA is $400 \mathrm{mg}$ (as $2 \times 200 \mathrm{mg}$ capsules) orally twice daily (this is without the loading dose used in the ENLIVEN study). If hepatotoxicity occurs, as demonstrated by elevations in liver enzyme tests, pexidartinib dosing should be withheld and permanently discontinued or restarted at a lower dosage per current labeling. The first dose reduction is to $600 \mathrm{mg}$ daily ( $200 \mathrm{mg}$ in the morning and $400 \mathrm{mg}$ in the evening), and the second is to $400 \mathrm{mg}$ daily (200 $\mathrm{mg}$ twice daily). Pexidartinib should be discontinued if patients are unable to tolerate $400 \mathrm{mg}$ daily [19]. 
Table 2. Treatment-emergent adverse events following long-term pexidartinib treatment across trials.

\begin{tabular}{|c|c|c|c|c|c|c|}
\hline \multirow[t]{2}{*}{$\begin{array}{l}\text { TEAEs, n (\%) })^{\ddagger} \\
\text { Skin disorders }\end{array}$} & \multicolumn{2}{|c|}{$\begin{array}{c}\text { ENLIVEN randomized } \\
\begin{array}{c}\left(1000 \mathrm{mg} / \text { day }^{\dagger}\right) \\
\mathrm{n}=61\end{array}\end{array}$} & \multicolumn{2}{|c|}{$\begin{array}{c}\text { ENLIVEN crossover } \\
\left(800 \mathrm{mg} / \text { day }^{\dagger}\right) \\
\mathrm{n}=30\end{array}$} & \multicolumn{2}{|c|}{$\begin{array}{l}\text { PLX108-01 TGCT cohort } \\
\begin{array}{c}\left(1000 \mathrm{mg} / \text { day }^{\dagger}\right) \\
\mathrm{n}=39\end{array}\end{array}$} \\
\hline & All grades & Grade $\geq 3$ & All grades & Grade $\geq 3$ & All grades & Grade $\geq 3$ \\
\hline Hair color changes & $45(74)$ & 0 & $25(83)$ & 0 & $28(72)$ & 0 \\
\hline Rash & $17(28)$ & $1(2)$ & $8(27)$ & 0 & $12(31)$ & 0 \\
\hline Pruitus & $10(16)$ & $1(2)$ & $6(20)$ & 0 & $14(36)$ & 0 \\
\hline Rash maculopapular & $10(16)$ & $1(2)$ & $3(10)$ & 0 & $8(21)$ & 0 \\
\hline Erythema & $2(3)$ & $1(2)$ & $6(20)$ & 0 & $8(21)$ & 0 \\
\hline Skin hypopigmentation & $5(8)$ & 0 & $1(3)$ & 0 & $7(18)$ & 0 \\
\hline \multicolumn{7}{|l|}{ General disorders } \\
\hline Fatigue & $35(57)$ & 0 & $8(27)$ & 0 & $36(92)$ & $1(3)$ \\
\hline Edema peripheral & $13(21)$ & 0 & $6(20)$ & 0 & $11(28)$ & 0 \\
\hline Face edema & $9(15)$ & $1(2)$ & $6(20)$ & $1(3)$ & $6(15)$ & 0 \\
\hline Asthenia & $9(15)$ & 0 & $7(23)$ & 0 & 0 & 0 \\
\hline \multicolumn{7}{|l|}{ Gastrointestinal disorders } \\
\hline Nausea & $28(46)$ & 0 & $7(23)$ & 0 & $26(67)$ & 0 \\
\hline Diarrhea & $18(30)$ & 0 & $9(30)$ & 0 & $15(38)$ & $3(8)$ \\
\hline Vomiting & $14(23)$ & $1(2)$ & $2(7)$ & 0 & $13(33)$ & $1(3)$ \\
\hline Constipation & $10(16)$ & 0 & $3(10)$ & 0 & $11(28)$ & 0 \\
\hline Abdominal pain & $15(25)$ & 0 & $3(10)$ & 0 & $2(5)$ & 0 \\
\hline Dry mouth & $8(13)$ & 0 & $4(13)$ & 0 & $4(10)$ & 0 \\
\hline \multicolumn{7}{|l|}{ Nervous disorders } \\
\hline Dysgeusia & $17(28)$ & 0 & $7(23)$ & 0 & $15(38)$ & 0 \\
\hline Headache & $14(23)$ & $1(2)$ & $6(20)$ & 0 & $13(33)$ & 0 \\
\hline Dizziness & $9(15)$ & $1(2)$ & $4(13)$ & 0 & $11(28)$ & 0 \\
\hline \multicolumn{7}{|l|}{ Musculoskeletal disorders } \\
\hline Arthralgia & $17(28)$ & $2(3)$ & $10(33)$ & 0 & $24(62)$ & $1(3)$ \\
\hline Pain in extremity & $7(11)$ & 0 & $4(13)$ & 0 & $10(26)$ & 0 \\
\hline \multicolumn{7}{|l|}{ Investigations } \\
\hline AST increased & $27(44)$ & $6(10)$ & $6(20)$ & $2(7)$ & $7(18)$ & $3(8)$ \\
\hline ALT increased & $19(31)$ & $6(10)$ & $7(23)$ & $3(10)$ & $7(18)$ & $4(10)$ \\
\hline Blood ALP increased & $9(15)$ & $4(7)$ & $1(3)$ & 0 & $4(10)$ & 0 \\
\hline \multicolumn{7}{|c|}{ Metabolic/nutrition disorders } \\
\hline Decreased appetite & $11(18)$ & 0 & $3(10)$ & 0 & $9(23)$ & 0 \\
\hline Hypophosphatemia & $3(5)$ & $2(3)$ & $2(7)$ & $1(3)$ & $11(28)$ & $5(13)$ \\
\hline \multicolumn{7}{|l|}{ Eye disorders } \\
\hline Periorbital edema & $17(28)$ & $1(2)$ & $5(17)$ & 0 & $15(38)$ & 0 \\
\hline \multicolumn{7}{|l|}{ Vascular disorders } \\
\hline Hypertension & $14(23)$ & $4(7)$ & $10(33)$ & $2(7)$ & $8(21)$ & 0 \\
\hline \multicolumn{7}{|l|}{ Blood disorders } \\
\hline Anemia & $6(10)$ & $1(2)$ & $1(3)$ & 0 & $9(23)$ & $1(3)$ \\
\hline \multicolumn{7}{|l|}{ Respiratory disorders } \\
\hline Cough & $4(7)$ & 0 & $4(13)$ & 0 & $8(21)$ & 0 \\
\hline \multicolumn{7}{|l|}{ Infections } \\
\hline URTI & $7(11)$ & 0 & $1(3)$ & 0 & $6(15)$ & 0 \\
\hline \multicolumn{7}{|l|}{ Psychiatric disorders } \\
\hline Insomnia & $3(5)$ & 0 & $3(10)$ & 0 & $7(18)$ & 0 \\
\hline $\begin{array}{l}\text { †Starting dose. } \\
\text { ¥Only preferred terms with } \\
\text { ( } n=130 ; \text { all grades). With } \\
\text { TEAE: Treatment-emergent }\end{array}$ & 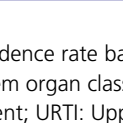 & 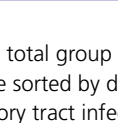 & $\begin{array}{l}\text { included. } \\
\text { quency of }\end{array}$ & $\begin{array}{l}\text { rted by desc } \\
\text { rm. }\end{array}$ & uency of syst & ass in the tot \\
\hline
\end{tabular}


Table 3. Registered pexidartinib trials in other clinical development areas.

\begin{tabular}{|c|c|c|c|}
\hline Identifier & Study design & Indication & Ref. \\
\hline $\begin{array}{l}\text { PIANO; } \\
\text { NCT02071940 }\end{array}$ & $\begin{array}{l}\text { Open-label, single-arm Phase II study of pexidartinib } \\
\text { monotherapy }\end{array}$ & Advanced KIT-mutated acral and mucosal melanoma & [48] \\
\hline NCT02777710 & Phase I study of pexidartinib in combination with durvalumab & Metastatic/advanced pancreatic or colorectal cancer & [49] \\
\hline NCT02390752 & Phase I/II study of pexidartinib monotherapy & $\begin{array}{l}\text { Children and young adults with refractory leukemias or refractory } \\
\text { solid tumors including neurofibromatosis type } 1 \text { associated plexiform } \\
\text { neurofibromas }\end{array}$ & {$[50]$} \\
\hline NCT01596751 & Phase Ib/Il study of pexidartinib in combination with eribulin & Metastatic breast cancer & {$[51]$} \\
\hline NCT01790503 & $\begin{array}{l}\text { Phase } \mathrm{lb} / \mathrm{Il} \text { study of pexidartinib in combination with radiation } \\
\text { therapy and temozolomide }\end{array}$ & Newly diagnosed glioblastoma & [52] \\
\hline NCT02584647 & Phase $1 /$ II study of pexidartinib in combination with sirolimus & Sarcoma and malignant peripheral nerve sheath tumors & [53] \\
\hline NCT02401815 & Phase I/II study of pexidartinib in combination with PLX9486 & Advanced solid tumors & [54] \\
\hline NCT03158103 & Phase I study of pexidartinib in combination with binimetinib & Gastrointestinal stromal tumors & [55] \\
\hline
\end{tabular}

Importantly, pexidartinib should be administered on an empty stomach, at least $1 \mathrm{~h}$ before or $2 \mathrm{~h}$ after a meal or snack [19]. Taking pexidartinib with a high-fat ( $\sim 50 \%$ of calories derived from fat) meal results in an approximate doubling of plasma exposure when compared with administering pexidartinib in a fasted state, while a low-fat ( $\sim 25 \%$ of calories derived from fat) meal increases pexidartinib exposure by approximately $60 \%$. The presence of food increases pexidartinib exposure which may lead to an increased risk of adverse events, including hepatotoxicity; therefore, patients must carefully abide by dosing guidelines as outlined in current prescribing information [19].

\section{Ongoing clinical studies}

In patients with TGCT, the Phase I (PLX108-01) and ENLIVEN pexidartinib studies are expected to continue for follow-up until 2021 [45,46]. A prospective observational registry study (NCT02948088) is following patients with D-TGCT to determine the economic impact, management and burden of TGCT [47]. Whether treatment interruptions after response are feasible will be the subject of these and other future postregistration studies. Clinical studies of pexidartinib in other tumor types have been conducted (Table 3). Pexidartinib is not currently approved for indications other than TGCT.

\section{Conclusion}

Based on the results from the ENLIVEN study, pexidartinib has been approved in the USA for adult patients with symptomatic TGCT associated with severe morbidity or functional limitations and not amenable to improvement with surgery. For these patients, no other approved treatment options exist; therefore, pexidartinib represents a valuable treatment option for selected patients with TGCT. Results from clinical studies demonstrate the efficacy of pexidartinib, which included tumor response as well as improved range of motion. Pexidartinib has generally manageable side effects in patients with TGCT, but it also causes serious hepatotoxicity that may be fatal; therefore, patients should be monitored closely for signs of liver toxicity, particularly during initial treatment, with prompt interruption or discontinuation of therapy if needed to mitigate the severity of the risk.

\section{Future perspective}

Until recently, there was no approved systemic therapy for patients with TGCT. Pexidartinib represents a significant advancement in the field, which in 5-10 years from now should result in the improvement in symptoms and physical functioning of many patients with TGCT who could not be treated successfully by surgical methods. Opportunities for future studies with pexidartinib include adjuvant treatment. 
- Tenosynovial giant cell tumor (TGCT) is a rare, nonmalignant neoplasm that can be locally aggressive.

- Patients with TGCT experience pain, impaired daily functioning and poorer quality of life compared with the general public.

- TGCT lesions are driven by an overexpression of CSF1 present in a minority of neoplastic cells, which leads to the accumulation of inflammatory cells.

- Pexidartinib is a CSF1 receptor (CSF1R) inhibitor approved in the USA for the treatment of adult patients with symptomatic TGCT associated with severe morbidity or functional limitations and not amenable to improvement with surgery.

- Pexidartinib is only available as part of a Risk Evaluation and Mitigation Strategy program; the current dosage is $400 \mathrm{mg}$ orally twice daily.

Pharmacodynamics \& pharmacokinetics

- Pexidartinib should be administered on an empty stomach, at least $1 \mathrm{~h}$ before or $2 \mathrm{~h}$ after a meal or snack.

- Taking pexidartinib with a high-fat ( $\sim 50 \%$ of calories derived from fat) meal results in an approximate doubling of plasma exposure when compared with administering pexidartinib in a fasted state, while a low-fat ( $25 \%$ of calories derived from fat) meal increases pexidartinib exposure by approximately $60 \%$.

- Pharmacokinetics modeling demonstrates no differences between Asian and non-Asian patients, or clinically meaningful effects of demographic characteristics or renal and hepatic function.

Clinical studies of pexidartinib in TGCT

- Pexidartinib demonstrates tumor responses in patients with TGCT: by RECIST, overall response rates in the Phase III ENLIVEN study were $39 \%$ at 25 weeks, $54 \%$ at 17 months and $60 \%$ at 19 months; by tumor volume score, a TGCT-specific measurement of tumor response, overall response rates were $56 \%$ at 25 weeks, $64 \%$ at 17 months and $65 \%$ at 19 months.

- Patients who received pexidartinib experienced improvements from baseline in range of motion $(p=0.0043)$ compared with placebo.

Safety

- Pexidartinib had a generally manageable safety profile in clinical trials, except for hepatotoxicity; in the ENLIVEN study, adverse events were reported in $98 \%$ of patients who received pexidartinib and $93 \%$ of patients who received placebo.

- The most common adverse events of any grade in ENLIVEN were hair color changes (67\%), fatigue (54\%), AST increase $(39 \%)$, nausea $(38 \%)$, ALT increase $(28 \%)$, dysgeusia $(25 \%)$, arthralgia $(23 \%)$, diarrhea $(20 \%)$ and vomiting $(20 \%)$.

- Grade 3 or 4 adverse events occurring at a higher rate in the pexidartinib group than in the placebo group were increased AST (10 vs $0 \%$ ), increased ALT (10 vs $0 \%$ ), ALP (7 vs $0 \%$ ) and hypertension ( 5 vs $0 \%$ ).

Hepatic adverse reactions

- In TGCT studies, five patients had serious hepatic adverse reactions, all within the first 2 months of treatment.

- All cases were reversible with discontinuation of pexidartinib, four resolved within 2 months and one case of ductopenia and severe cholestasis resolved after 7 months.

- Across the clinical program, there were two irreversible cases of cholestatic liver injury. One patient died with advanced cancer and ongoing liver toxicity and one patient required a liver transplant.

Clinical approach to managing pexidartinib safety

- Careful monitoring of liver function is advised, particularly in the first 2 months of treatment.

- The presence of food increases pexidartinib exposure which may lead to an increased risk of adverse events, including hepatotoxicity; therefore, patients must closely follow labeling guidance for dosing, remembering that pexidartinib should be taken on an empty stomach at least $1 \mathrm{~h}$ before or $2 \mathrm{~h}$ after a meal or snack.

Conclusion

- Pexidartinib is the only approved systemic treatment for adult patients with symptomatic TGCT associated with severe morbidity or functional limitations and not amenable to improvement with surgery, for whom no other approved treatment options exist.

\section{Author contributions}

Both authors contributed to the drafting and critical revision of this manuscript and gave final approval to submit it for publication.

Financial \& competing interests disclosure

H Gelderblom: research grant to institution from Daiichi Sankyo, Inc. M van de Sande: research grant to institution from Daiichi Sankyo, Inc. The authors have no other relevant affiliations or financial involvement with any organization or entity with a financial interest in or financial conflict with the subject matter or materials discussed in the manuscript apart from those disclosed. 
Medical writing and editorial assistance were provided by M Tradewell and H Nyce of SciFluent Communications, Inc., and were financially supported by Daiichi Sankyo.

Open access

This work is licensed under the Attribution-NonCommercial-NoDerivatives 4.0 Unported License. To view a copy of this license, visit http://creativecommons.org/licenses/by-nc-nd/4.0/

\section{References}

Papers of special note have been highlighted as: $\bullet$ of interest; $\bullet \bullet$ of considerable interest

1. de Saint Aubain Somerhausen N, van de Rijn M. Tenosynovial giant cell tumour: localized type, diffuse type. In: World Health Organization Classification of Tumours of Soft Tissue and Bone. Fletcher CDM, Bridge JA, Hogendoorn PCW, Martens F. (Eds). 100-103 IARC Press, Lyon, France (2013).

2. Myers BW, Masi AT. Pigmented villonodular synovitis and tenosynovitis: a clinical epidemiologic study of 166 cases and literature review. Medicine (Baltimore) 59(3), 223-238 (1980).

3. Mastboom MJL, Verspoor FGM, Verschoor AJ et al. Higher incidence rates than previously known in tenosynovial giant cell tumors. Acta. Orthop. 88(6), 688-694 (2017).

- Highlights tenosynovial giant cell tumor (TGCT) incidence rates.

4. Ehrenstein V, Andersen SL, Qazi I et al. Tenosynovial giant cell tumor: incidence, prevalence, patient characteristics, and recurrence. A registry-based cohort study in Denmark. J. Rheumatol. 44(10), 1476-1483 (2017).

5. Staals EL, Ferrari S, Donati DM, Palmerini E. Diffuse-type tenosynovial giant cell tumour: current treatment concepts and future perspectives. Eur. J. Cancer 63, 34-40 (2016).

6. Brahmi M, Vinceneux A, Cassier PA. Current systemic treatment options for tenosynovial giant cell tumor/pigmented villonodular synovitis: targeting the CSF1/CSF1R axis. Curr. Treat. Options Oncol. 17(2), 10 (2016).

7. Mastboom MJ, Planje R, van de Sande MA. The patient perspective on the impact of tenosynovial giant cell tumors on daily living: crowdsourcing study on physical function and quality of life. Interact. J. Med. Res. 7(1), e4 (2018).

- Evaluates the impact of TGCT on patient physical function and daily activities.

8. Lopez-Bastida J, Ye X, Laeis P et al. Health-related quality of life in tenosynovial giant cell tumor (TGCT) patients in Europe and US: an observational disease registry. CTOS 22, S866 (2019).

9. von Mehren M, Kane JMI, Benjamin RS et al. NCCN Clinical Practice Guidelines in Soft Tissue Sarcoma. Version 4.2019 (2019). www.nccn.org/professionals/physician_gls/pdf/sarcoma.pdf

10. Dangoor A, Seddon B, Gerrand C, Grimer R, Whelan J, Judson I. UK guidelines for the management of soft tissue sarcomas. Clin. Sarcoma Res. 6, 20 (2016).

11. Casali PG, Abecassis N, Aro HT et al. Soft tissue and visceral sarcomas: ESMO-EURACAN Clinical Practice Guidelines for diagnosis, treatment and follow-up. Ann. Oncol. 29(Suppl. 4), iv268-iv269 (2018).

12. Gelhorn HL, Tong S, McQuarrie K et al. Patient-reported symptoms of tenosynovial giant cell tumors. Clin. Ther. 38(4), 778-793 (2016).

- Describes patient-reported symptoms of TGCT and identifies patient-reported outcomes instruments for assessing the effects of TGCT treatments.

13. Ravi V, Wang WL, Lewis VO. Treatment of tenosynovial giant cell tumor and pigmented villonodular synovitis. Curr. Opin. Oncol. 23(4), 361-366 (2011).

14. Tap WD, Wainberg ZA, Anthony SP et al. Structure-guided blockade of CSF1R kinase in tenosynovial giant-cell tumor. N. Engl. J. Med. 373(5), 428-437 (2015).

-. Reports results from a Phase I trial of pexidartinib in patients with TGCT.

15. Cassier PA, Gelderblom H, Stacchiotti S et al. Efficacy of imatinib mesylate for the treatment of locally advanced and/or metastatic tenosynovial giant cell tumor/pigmented villonodular synovitis. Cancer 118(6), 1649-1655 (2012).

16. Gelderblom $\mathrm{H}$, Cropet $\mathrm{C}$, Chevreau $\mathrm{C}$ et al. Nilotinib in locally advanced pigmented villonodular synovitis: a multicentre, open-label, single-arm, Phase 2 trial. Lancet Oncol. 19(5), 639-648 (2018).

17. Mastboom MJL, Palmerini E, Verspoor FGM et al. Surgical outcomes of patients with diffuse-type tenosynovial giant-cell tumours: an international, retrospective, cohort study. Lancet Oncol. 20(6), 877-886 (2019).

-. Provides a comprehensive overview of TGCT and assesses the clinical profile and management of the disease.

18. Lin F, Qian C, Kwong J. Treatment patterns of tenosynovial giant cell tumor among commercially insured patients: a retrospective claims analysis. J. Clin. Oncol. 36(Suppl. 15), e18737-e18737 (2018).

19. Daiichi-Sankyo Company Limited. TURALIO ${ }^{T M}$ full prescribing information (2020). https://dsi.com/prescribing-information-portlet/getPIContent?productName=Turalio\&inline=true 
20. West RB, Rubin BP, Miller MA et al. A landscape effect in tenosynovial giant-cell tumor from activation of CSF1 expression by a translocation in a minority of tumor cells. Proc Natl Acad. Sci. USA 103(3), 690-695 (2006).

21. Patwardhan PP, Surriga O, Beckman MJ et al. Sustained inhibition of receptor tyrosine kinases and macrophage depletion by PLX3397 and rapamycin as a potential new approach for the treatment of MPNSTs. Clin. Cancer Res. 20(12), 3146-3158 (2014).

22. Ao JY, Zhu XD, Chai ZT et al. Colony-stimulating factor 1 receptor blockade inhibits tumor growth by altering the polarization of tumor-associated macrophages in hepatocellular carcinoma. Mol. Cancer Ther. 16(8), 1544-1554 (2017).

23. Yin O FJ, Polhamus D, Zahir $\mathrm{H}$ et al. Exposure-response analysis of efficacy and safety for pexidartinib in patients with tenosynovial giant cell tumor (TGCT). Presented at: Tenth American Conference on Pharmacometrics (ACoP10). FL, USA (2019). Poster W-017.

24. Yin O, Kang J, Knebel W et al. Population pharmacokinetic analysis of pexidartinib in healthy subjects and patients with tenosynovial giant cell tumor (TGCT) or other solid tumors. Presented at: Tenth American Conference on Pharmacometrics (ACoP10). FL, USA (2019). Poster M-028.

25. Lin C-C, Guo J, Tap WD et al. Population pharmacokinetic analysis for comparison of pexidartinib exposure in Asian and non-Asian patients. Presented at: 2019 Connective Tissue Oncology Society (CTOS) Annual Meeting. Tokyo, Japan (2019). Abstract 3253949.

26. Peterfy C, Tap WD, DiCarlo J et al. MRI assessment of oral CSF1 receptor inhibition with PLX3397 for tenosynovial giant cell tumor using novel modified RECIST, tumor volume scoring and tissue damage scoring methods. Presented at: Orthopaedic Research Society Annual Meeting 2015. NV, USA (2015). Poster 2028.

27. Tap WD, Gelderblom H, Palmerini E et al. Pexidartinib versus placebo for advanced tenosynovial giant cell tumour (ENLIVEN): a randomised Phase 3 trial. Lancet 394(10197), 478-487 (2019).

-• Reports results from the Phase III registrational study of pexidartinib in patients with TGCT.

28. Tap WD, Gelhorn HL, Ye X et al. Improvement in patient-reported pain in a Phase iii trial of pexidartinib (PLX3397) among patients with tenosynovial giant cell tumor (TGCT). Presented at: 2019 Connective Tissue Oncology Society (CTOS) Annual Meeting. Tokyo, Japan (2019).

29. van de Sande M, Staals E, Gronchi A et al. Pexidartinib for advanced tenosynovial giant cell tumor: patient subgroup results from the Phase 3 ENLIVEN study. Presented at: 2019 European Musculo-Skeletal Oncology Society (EMSOS) Annual Meeting. Florence, Italy (2019). Abstract 2250 .

30. Gelderblom H, Tap WD, Palmerini E et al. Pexidartinib for advanced tenosynovial giant cell tumor (TGCT): long-term efficacy and safety from the Phase 3 ENLIVEN and Phase 1 PLX108-01 (TGCT cohort) studies. Presented at: 2019 American Society of Clinical Oncology (ASCO) Annual Meeting. IL, USA (2019). Abstract 11042.

31. Healey JH, Gelderblom H, Wagner AJ et al. Pexidartinib for locally advanced tenosynovial giant cell tumor (TGCT): overall long-term pooled efficacy and safety with characterization of hepatic adverse reactions (ARs) from ENLIVEN and other studies. Presented at: 2019 Musculo Skeletal Tumor Society (MSTS) Annual Meeting. OR, USA (2019). Presentation 46.

32. Healey JH, Gelderblom H, Wagner AJ et al. Pexidartinib for locally advanced tenosynovial giant cell tumor: overall long-term pooled efficacy and safety with characterization of hepatic adverse reactions from ENLIVEN and other studies. Presented at: 2019 Connective Tissue Oncology Society (CTOS) Annual Meeting. Tokyo, Japan (2019). Abstract 3208650.

33. Butowski N, Colman H, De Groot JF et al. Orally administered colony stimulating factor 1 receptor inhibitor PLX3397 in recurrent glioblastoma: an Ivy Foundation Early Phase Clinical Trials Consortium Phase II study. Neuro. Oncol. 18(4), 557-564 (2016).

34. Colman H, Raizer JJ, Walbert $\mathrm{T}$ et al. Phase $1 \mathrm{~b} / 2$ study of pexidartinib (PEX) in combination with radiation therapy (XRT) and temozolomide (TMZ) in newly diagnosed glioblastoma. J. Clin. Oncol. 36(Suppl. 15), 2015-2015 (2018).

35. Wesolowski R, Sharma N, Reebel L et al. Phase Ib study of the combination of pexidartinib (PLX3397), a CSF-1R inhibitor, and paclitaxel in patients with advanced solid tumors. Ther. Adv. Med. Oncol. 11, 1758835919854238 (2019).

36. Manji GA, Tine BAV, Lee SM et al. Phase 1 combination therapy with pexidartinib (PEX) and sirolimus (S) to target tumor-associated macrophages in pigmented villonodular synovitis, malignant peripheral nerve sheath tumors, and other soft tissue sarcomas. J. Clin. Oncol. 37(Suppl. 15), 11055-11055 (2019).

37. Rosenbaum E, Kelly C, D’Angelo SP et al. A Phase I study of binimetinib (MEK162) combined with pexidartinib (PLX3397) in patients with advanced gastrointestinal stromal tumor. Oncologist 24(10), e1309-e1983 (2019).

38. Wagner AJ TW, Shields AF, Patnaik A et al. A Phase I pharmacokinetic (PK) and pharmacodynamic (PD) study of PLX9486 alone and in combination (combo) with the KIT inhibitors pexidartinib (pexi) or sunitinib (su) in patients (Pts) with advanced solid tumors and gastrointestinal stromal tumor (GIST). J. Clin. Oncol. 36(Suppl.15), 11509 (2018).

39. Hittson L, Glod J, Amaya M, Derdak J, Widemann BC, Kaplan R. Phase I study of pexidartinib (PLX3397) in children with refractory leukemias and solid tumors including neurofibromatosis type I (NF1) related plexiform neurofibromas (PN). J. Clin. Oncol. 35(Suppl. 15), 10546-10546 (2017).

40. Zahir H, Darpo B, Gu X et al. A concentration-QTc analysis to determine the effect of pexidartinib on the QTc interval. Presented at: 2019 American College of Clinical Pharmacology (ACCP) Annual Meeting. IL, USA (2019).Poster 073.

41. Bauer S, Lewis JH HG, van de Sande M et al. Pexidartinib (Pex) for locally advanced tenosynovial giant cell tumour (TGCT): characterization of hepatic adverse reactions (ARs). Presented at: ESMO Congress 2019. Barcelona, Spain (2019). Poster 1696P. 
42. Radi ZA, Koza-Taylor PH, Bell RR et al. Increased serum enzyme levels associated with kupffer cell reduction with no signs of hepatic or skeletal muscle injury. Am. J. Pathol. 179(1), 240-247 (2011).

43. Cannarile MA, Weisser M, Jacob W, Jegg AM, Ries CH, Ruttinger D. Colony-stimulating factor 1 receptor (CSF1R) inhibitors in cancer therapy. J. Immunother. Cancer 5(1), 53 (2017).

44. Piawah S, Hyland C, Umetsu SE, Esserman LJ, Rugo HS, Chien AJ. A case report of vanishing bile duct syndrome after exposure to pexidartinib (PLX3397) and paclitaxel. NPJ Breast Cancer 5, 17 (2019).

45. ClinicalTrials.gov. A Phase I study to assess safety, pharmacokinetics, and pharmacodynamics of PLX3397 in patients with advanced, incurable, solid tumors in which the target kinases are linked to disease pathophysiology. ClinicalTrials.gov, number NCT01004861. https://clinicaltrials.gov/ct2/show/NCT01004861

46. ClinicalTrials.gov. Phase 3 study of pexidartinib for pigmented villonodular synovitis (PVNS) or giant cell tumor of the tendon sheath (GCT-TS) (ENLIVEN). ClinicalTrials.gov, number NCT02371369. https://clinicaltrials.gov/ct2/show/NCT02371369

47. ClinicalTrials.gov. Tenosynovial giant cell tumors (TGCT) observational platform project (TOP). ClinicalTrials.gov, number NCT02948088. https:/clinicaltrials.gov/ct2/show/NCT02948088

48. ClinicalTrials.gov. PLX3397 KIT in Acral aNd mucOsal Melanoma (PIANO). ClinicalTrials.gov, number NCT02071940. https://clinicaltrials.gov/ct2/show/NCT02071940

49. ClinicalTrials.gov. Evaluation of safety and activity of an anti-PDL1 antibody (DURVALUMAB) combined with CSF-1R TKI (PEXIDARTINIB) in patients with metastatic/advanced pancreatic or colorectal cancers (MEDIPLEX). ClinicalTrials.gov, number NCT02777710. https://clinicaltrials.gov/ct2/show/NCT02777710

50. ClinicalTrials.gov. PLX3397 in children and young adults with refractory leukemias and refractory solid tumors including neurofibromatosis type 1 (NF1) associated plexiform neurofibromas (PN). ClinicalTrials.gov, number NCT02390752. https://clinicaltrials.gov/ct2/show/NCT02390752

51. ClinicalTrials.gov. Phase Ib/II study of PLX 3397 and eribulin in patients with metastatic breast cancer. ClinicalTrials.gov, number NCT01596751. https://clinicaltrials.gov/ct2/show/NCT01596751

52. ClinicalTrials.gov. A Phase $1 \mathrm{~b} / 2$ study of PLX3397 + radiation therapy + temozolomide in patients with newly diagnosed glioblastoma. ClinicalTrials.gov, number NCT01790503. https://clinicaltrials.gov/ct2/show/study/NCT01790503

53. ClinicalTrials.gov. PLX3397 plus sirolimus in unresectable sarcoma and malignant peripheral nerve sheath tumors (PLX3397). ClinicalTrials.gov, number NCT02584647. https://clinicaltrials.gov/ct2/show/NCT02584647

54. ClinicalTrials.gov. PLX9486 as a single agent and in combination with PLX3397 or PLX9486 with sunitinib in patients with advanced solid tumors. ClinicalTrials.gov, number NCT02401815. https://clinicaltrials.gov/ct2/show/NCT02401815

55. ClinicalTrials.gov. A study of MEK162 (Binimetinib) in combination with pexidartinib in patients with advanced gastrointestinal stromal tumor (GIST). ClinicalTrials.gov, number NCT03158103. https://clinicaltrials.gov/ct2/show/NCT03158103 